$\pm N / E$

Global Journals Inc.

sits 3

Global JOURnAl of COMPUTER SCIENCE AND TECHNOlOGY: G

INTERDISCIPLINARY

Volume 20 Issue 2 Version 1.0 Year 2020

Type: Double Blind Peer Reviewed International Research Journal

Publisher: Global Journals

Online ISSN: 0975-4172 \& Print ISSN: 0975-4350

\title{
Performance Comparison of EIGRP, OSPF and RIP Routing Protocols using Cisco Packet Tracer and OPNET Simulator
}

By Md. Anwar Hossain, Md. Mohon Ali, Mst. Sharmin Akter \& Md.Shahriar Alam Sajib

Pabna University of Science and Technology

Abstract- In this paper, the intention has to create a network configuration that is similar for all routing protocols RIP, OSPF, and EIGRP by which we want to analysis the performance of these protocols using Cisco Packet Tracer and OPNET simulator. We use various protocols for forwarding the packets in a network topology. For successful delivery of the packets from the source node to the accurate destination node, the routers maintain a routing table. The amount of network information stored by a router depends on its algorithm. For the performance measure, we will simulate real-time scenarios of the networks using Cisco Packet Tracer and OPNET simulation tools. We will evaluate the performance of EIGRP, OSPF, and RIP based on of network convergence, Ethernet delay, security, and bandwidth requirement, etc. We will observe that the EIGRP routing protocol has the maximum link utilization followed by OSPF, and RIP routing protocols.

Keywords: routing protocol, EIGRP, OSPF, RIP, packet tracer, OPNET.

GJCST-G Classification: C.2.M

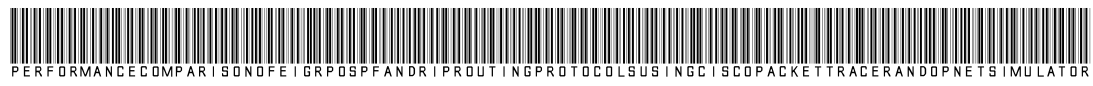

Strictly as per the compliance and regulations of:

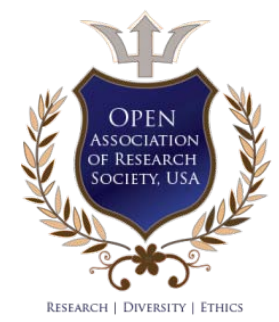

(c) 2020. Md. Anwar Hossain, Md. Mohon Ali, Mst. Sharmin Akter \& Md.Shahriar Alam Sajib. This is a research/review paper, distributed under the terms of the Creative Commons Attribution-Noncommercial 3.0 Unported License http://creativecommons.org/licenses/by-nc/3.0/), permitting all non-commercial use, distribution, and reproduction in any medium, provided the original work is properly cited. 


\title{
Performance Comparison of EIGRP, OSPF and RIP Routing Protocols using Cisco Packet Tracer and OPNET Simulator
}

\author{
Md. Anwar Hossain ${ }^{\alpha}$, Md. Mohon Ali ${ }^{\sigma}$, Mst. Sharmin Akter ${ }^{\rho}$ \& Md.Shahriar Alam Sajib ${ }^{\omega}$
}

\begin{abstract}
In this paper, the intention has to create a network configuration that is similar for all routing protocols RIP, OSPF, and EIGRP by which we want to analysis the performance of these protocols using Cisco Packet Tracer and OPNET simulator. We use various protocols for forwarding the packets in a network topology. For successful delivery of the packets from the source node to the accurate destination node, the routers maintain a routing table. The amount of network information stored by a router depends on its algorithm. For the performance measure, we will simulate real-time scenarios of the networks using Cisco Packet Tracer and OPNET simulation tools. We will evaluate the performance of EIGRP, OSPF, and RIP based on of network convergence, Ethernet delay, security, and bandwidth requirement, etc. We will observe that the EIGRP routing protocol has the maximum link utilization followed by OSPF, and RIP routing protocols.
\end{abstract}

Keywords: routing protocol, EIGRP, OSPF, RIP, packet tracer, OPNET.

\section{InTRODUCTION}

A routing protocol operates at layer three of the Open System Interconnection model. There are different types of routing protocols widely used in the network. EIGRP is a Cisco proprietary distancevector protocol based on the Diffusing Update Algorithm (DUAL). EIGRP only supports Cisco product. However, the convergence time of EIGRP is faster than other protocols and easy to configure.

In contrast, OSPF is a link-state interior gateway protocol based on the Dijkstra algorithm (Shortest Path First Algorithm). OSPF routing protocol has difficulty to configure network and high memory requirements. Our goal is to implement the routing protocols and compare the performance using Packet Tracer and OPNET. In this paper, we consider three routing protocols: EIGRP, OSPF, and RIP with real time applications. Our research question is; how well EIGRP over OSPF and RIP performs for real time applications?

Author a: Assistant Professor, Department of Information and Communication Engineering, Faculty of Engineering and Technology, Pabna University of Science and Technology, Pabna, Bangladesh. e-mail:manwar.ice@gmail.com

Author o: Student, Department of Information and Communication Engineering, Faculty of Engineering and Technology, Pabna University of Science and Technology, Pabna, Bangladesh.

e-mails: mohonali22@gmail.com, sharminputulsharmin@gmail.com, sajibpust130639@gmail.com

\section{il. Routing Protocol Overview}

\section{a) EIGRP}

Enhanced Interior Gateway Routing Protocol (EIGRP) is an interior gateway protocol suited for many different topology and media. In a well-designed network, EIGRP scales well and provides extremely rapid convergence times with minimal network traffic. EIGRP is an enhanced distance vector protocol, relying on the Diffused Update Algorithm (DUAL) to calculate the shortest path to a destination within a network.

b) OSPF

It is an Intra-domain routing protocol based on link state routing. Its domain is also an autonomous system. OSPF divides the independent system into different areas. Each area has an area boundary router, and all the routers in the area are connected to this. There is a backbone which consists of backbone routers. These backbone routers connect to the area boundary routers and facilitate communication. Then these routers connect the AS boundary routers which act as gateways.

c) RIP

The Routing Information Protocol, or RIP, is one of the most enduring of all routing protocols. RIP has four basic components: routing update process, RIP routing metrics, routing stability, and routing timers. Devices that support RIP send routing update messages at regular intervals and when the network topology changes. These RIP packets contain information about the networks that the devices can reach, as well as the number of routers or gateways that a packet must travel through the destination address.

\section{ili. Implementation using Packet Tracer}

Now we design and implement the routing protocol using Packet Tracer. We design a topology in the workspace. Then we implement each protocol in the network independently. The following figures show the physical topology of EIGRP, OSPF, and RIP. 


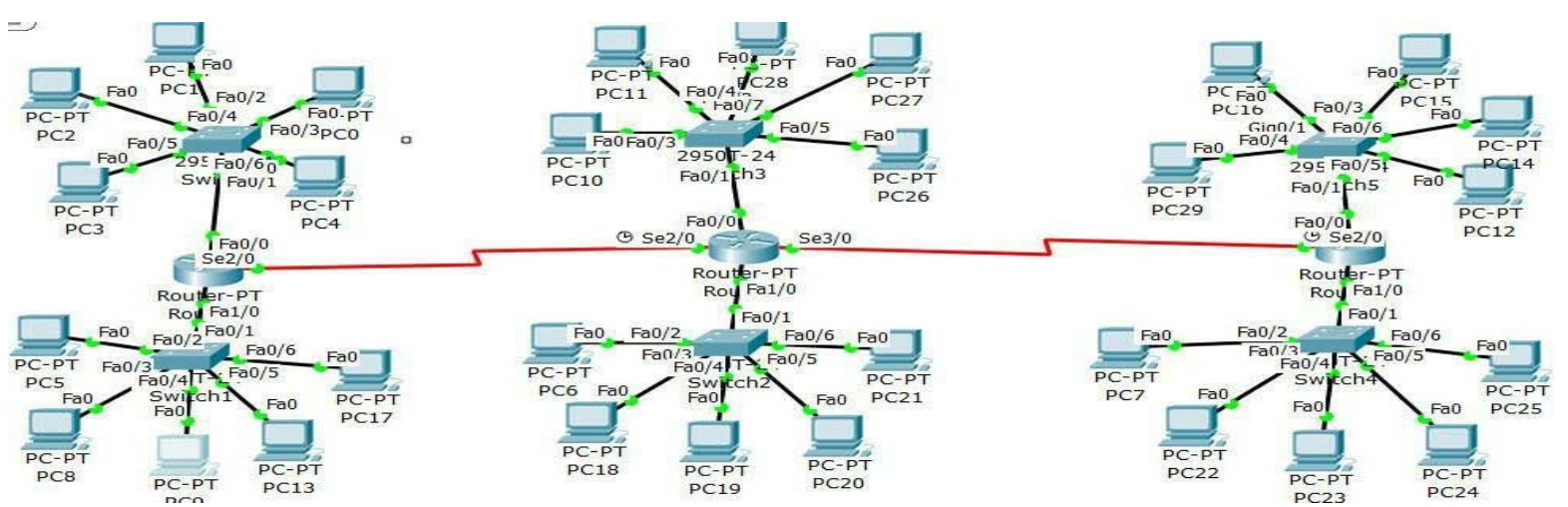

Fig.1: Network diagram of EIGRP
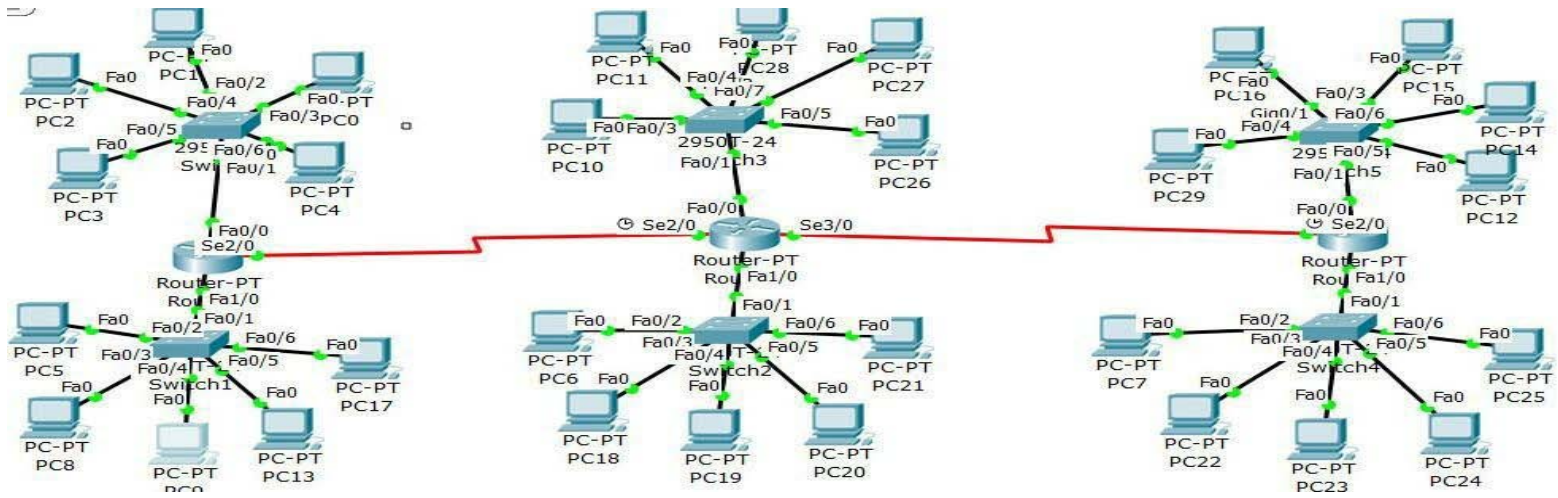

Fig.2: Network diagram of OSPF
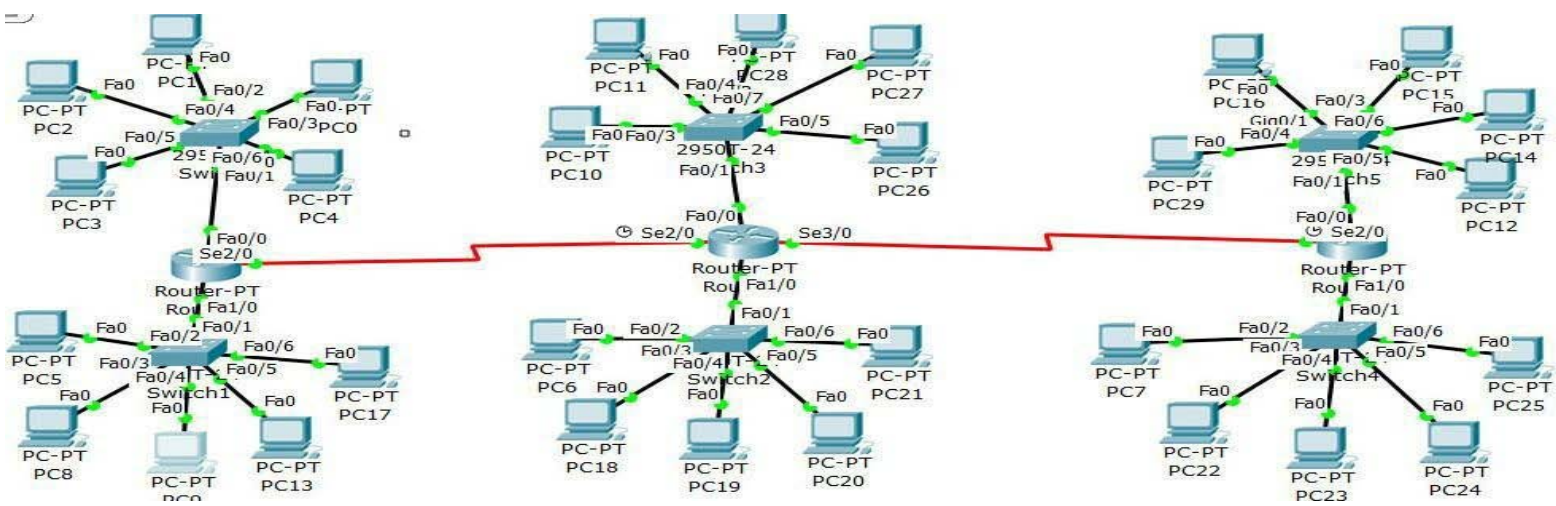

Fig.3: Network diagram of RIP

Fig.1 shows the topology of EIGRP. There are three routers, and each router contains two switches, and each switch comprises five end devices. Each end device encompasses a specific IP address, subnet mask, and a default gateway.

Fig.2 shows the topology of OSPF. There are three routers, and each router contains two switches, and each switch comprises five end devices. Each end device has specific IP address, subnet mask, and a default gateway.

Fig. 3 shows the topology of RIP. There are three routers, and each router contains two switches, and each switch comprises five end devices. Each end device encompasses a specific IP address, subnet mask, and a default gateway.

\section{iv. Opnet Simulation}

\section{a) Simulator}

The simulator can help to show the eventual real behavior of the selected system model. For performance optimization based on creating a model of the system to gain insight into their functioning. It is very easy to predict the estimation, and assumption of the real system by using simulation results.

We use Optimized Network Engineering Tools (OPNET) modeler as a simulation environment. OPNET is a simulator built on to Discrete Event System (DES), and it simulates the system behavior by modeling each event in the system and processes it through user defined processes[4].OPNET is very dominant software 
to simulate a heterogeneous network with various protocols.

\section{b) Design and Simulation in OPNET}

To simulate any network in OPNET, one should follow some steps one after another. Simulation in OPNET is very tranquil and user-friendly. The following figure shows the design and simulation steps in OPNET.

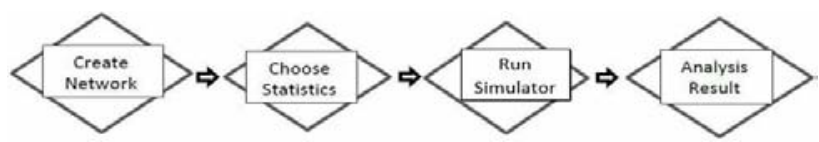

Fig.4: Designing steps

Fig. 5 shows the network topology in OPNET. In our experiment, we have created three scenarios that consist of twenty-five inter connected subnets.

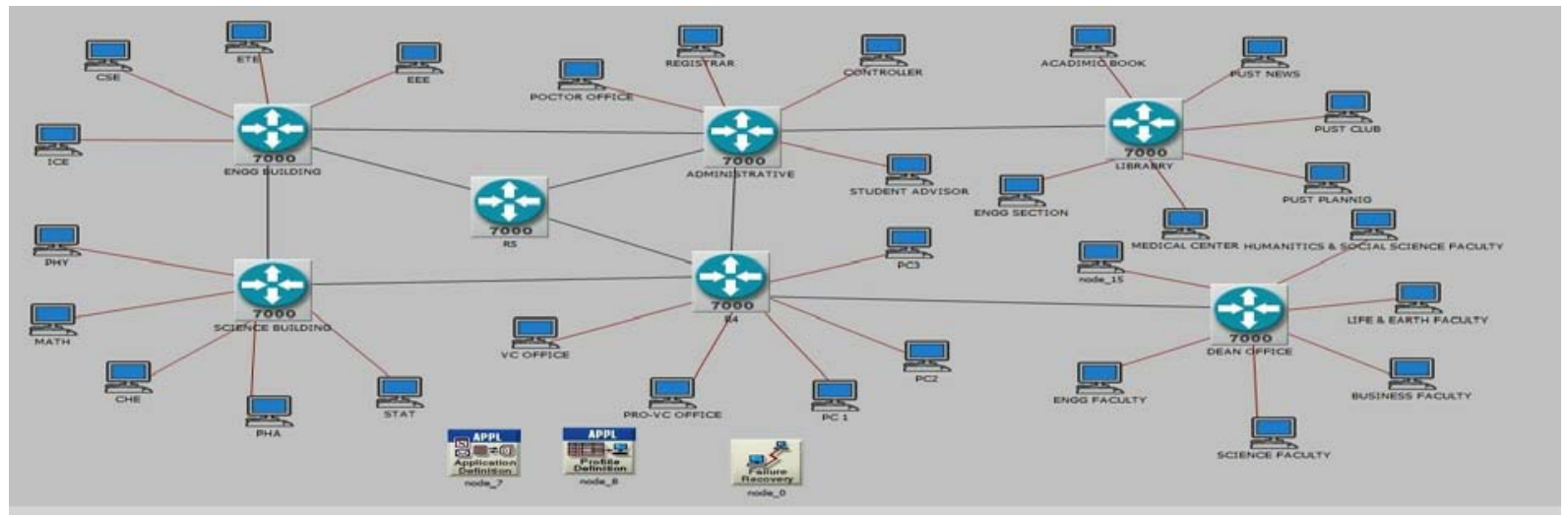

Fig.5: Network topology in OPNET

We have configured routers within each subnet by using EIGRP, OSPF and RIP routing protocols.

The network topology contains the following network devices and configuration utilities:CS 7200 Cisco Routers, Ethernet Server, Switch, PPP DS3 Duplex Link, PPP_DS1 Duplex Link, Ethernet 100 Base T Duplex Link, Ethernet Workstation, twenty-five Subnets. We connect the routers using PPP_DS3 duplex link with each other. We connect the switches to routers using the same duplex link and Ethernet workstations to switch using 10 Base T duplex links.

\section{c) Simulation Setup}

In the simulation arrangement for Application Definition, we add an Application Definition Object from the object palette into the workspace. Fig.6 shows the setup. The Application Configuration allows for generating different types of application traffic. As far as we concern real-time applications in our work, we set the Application Definition Object to support Video Streaming (Light).

In the simulation setup for Profile Definition, we add a Profile Configuration from the object palette into the workspace.Fig.6 shows the setup. A Profile Definition Object defines the profiles within the distinct application traffic of the Application Definition Objects. In the Profile Configuration, we create one profile. The Profile has the application support of Video Streaming (Light).

In the simulation setup for Failure/Recovery Configuration, we configure the failure link in the scenarios. The Failure events introduce disturbances in the routing topology, leading to additional intervals of convergence activity. The link connected between the
Director and the Engineering router is set to be failure and recover and time is in Table-1.Fig. 8 shows the Failure/Recovery configuration.

Table 1: Failure/Recovery time

\begin{tabular}{|c|c|}
\hline Status & Time (second) \\
\hline Fail & 240 \\
\hline Recover & 420 \\
\hline Fail & 520 \\
\hline Recover & 580 \\
\hline Fail & 610 \\
\hline Recover & 620 \\
\hline Fail & 625 \\
\hline Recover & 626 \\
\hline Fail & 726 \\
\hline Recover & 826 \\
\hline
\end{tabular}

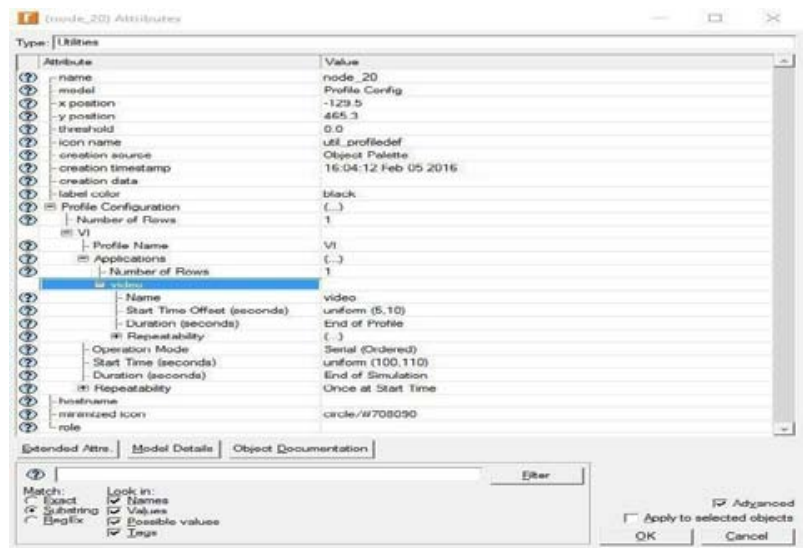

Fig.6: Application definition configuration 


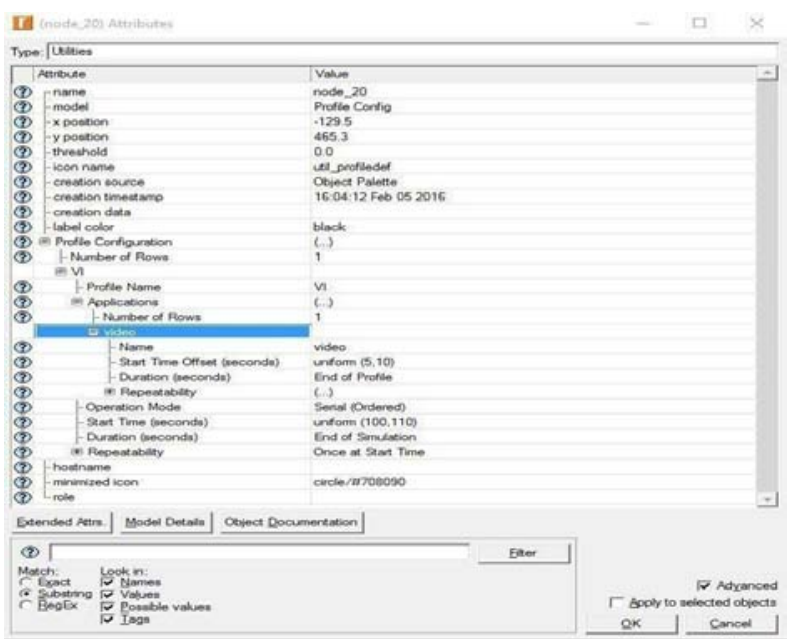

Fig.7: Profile definition configuration

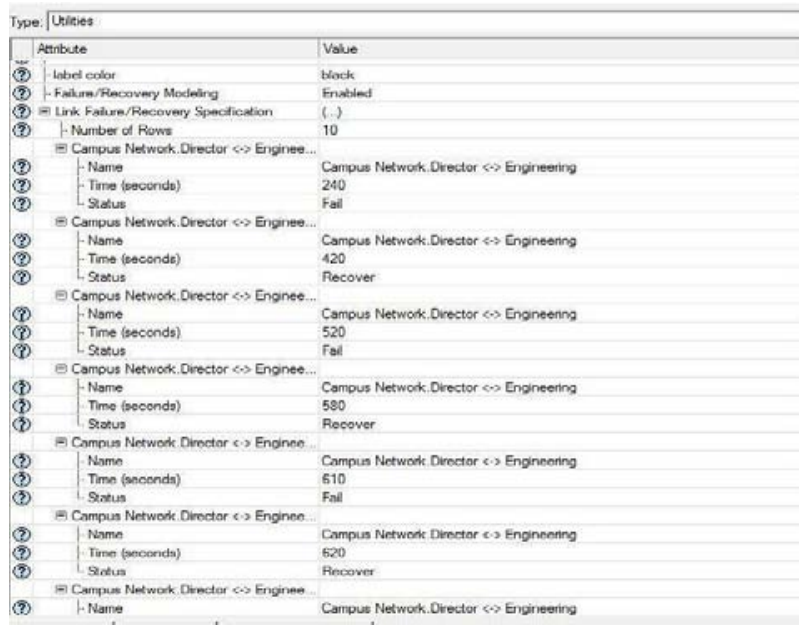

Fig.8: Failure/Recovery configuration

\section{d) Setup for Individual DES statistics}

Since we will examine three protocols, we set the individual statistics differently. It concludes that we can use the features for comparison. The following figures show the configuration of Convergence Activity, Convergence Duration, and Traffic Sent (bits/sec).
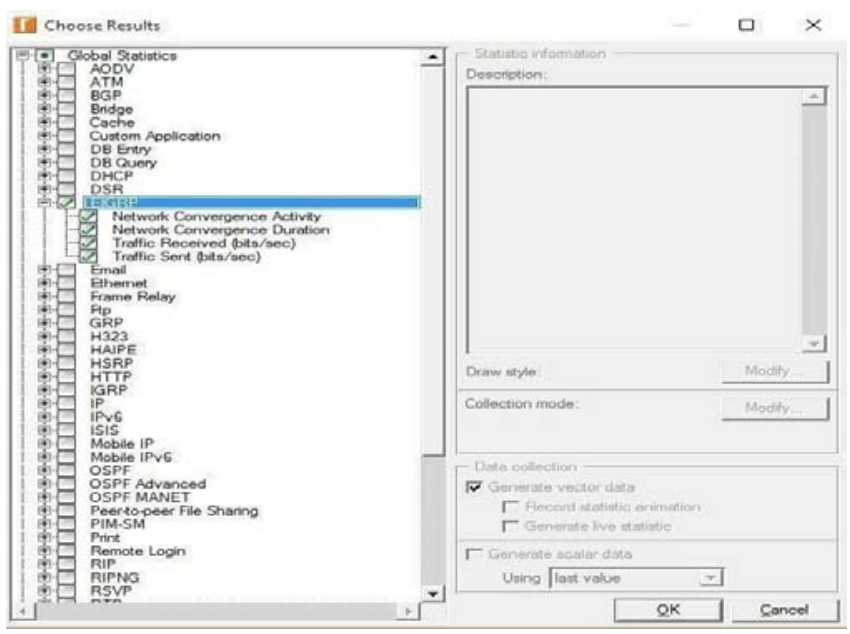

Fig.9: EIGRP DES statistics

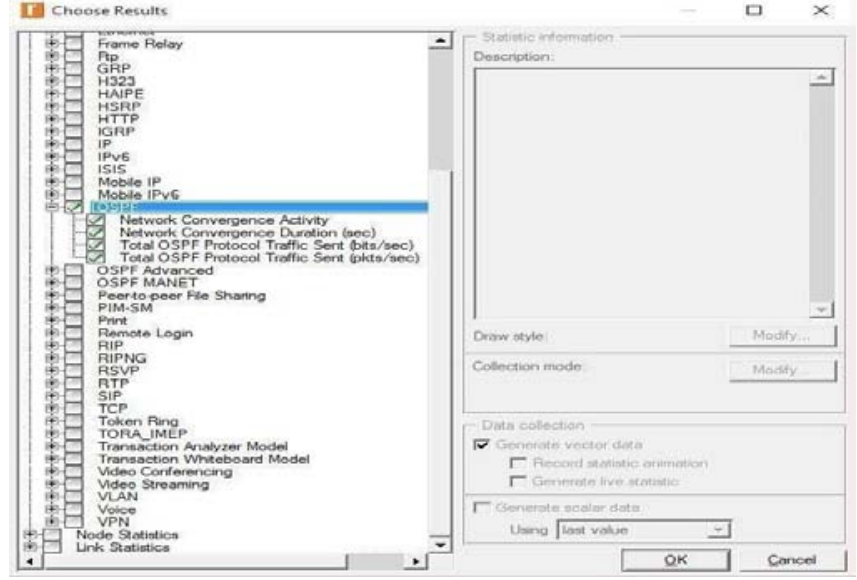

Fig.10: OSPF DES statistics

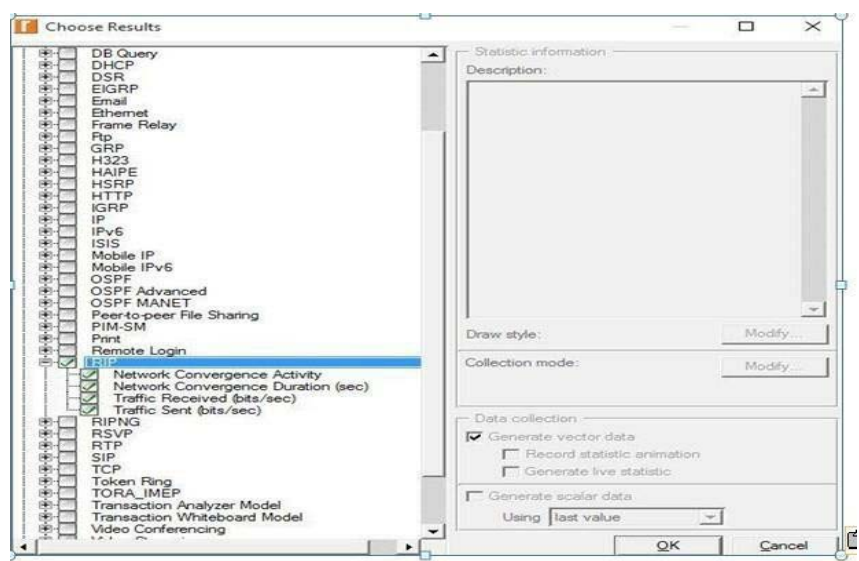

Fig.11: RIP DES statistics

\section{e) Scenario}

Each routing protocol implemented in the same topology has a different scenario. The following figures show the scenario of each routing protocol separately. 


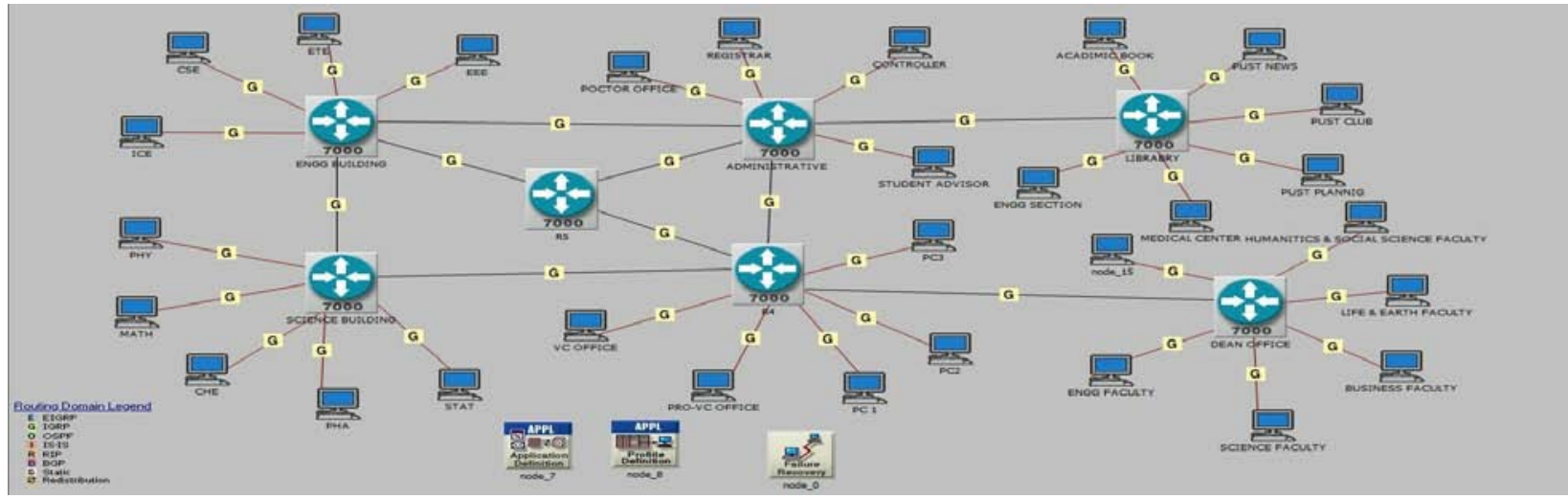

Fig. 12: EIGRP scenario

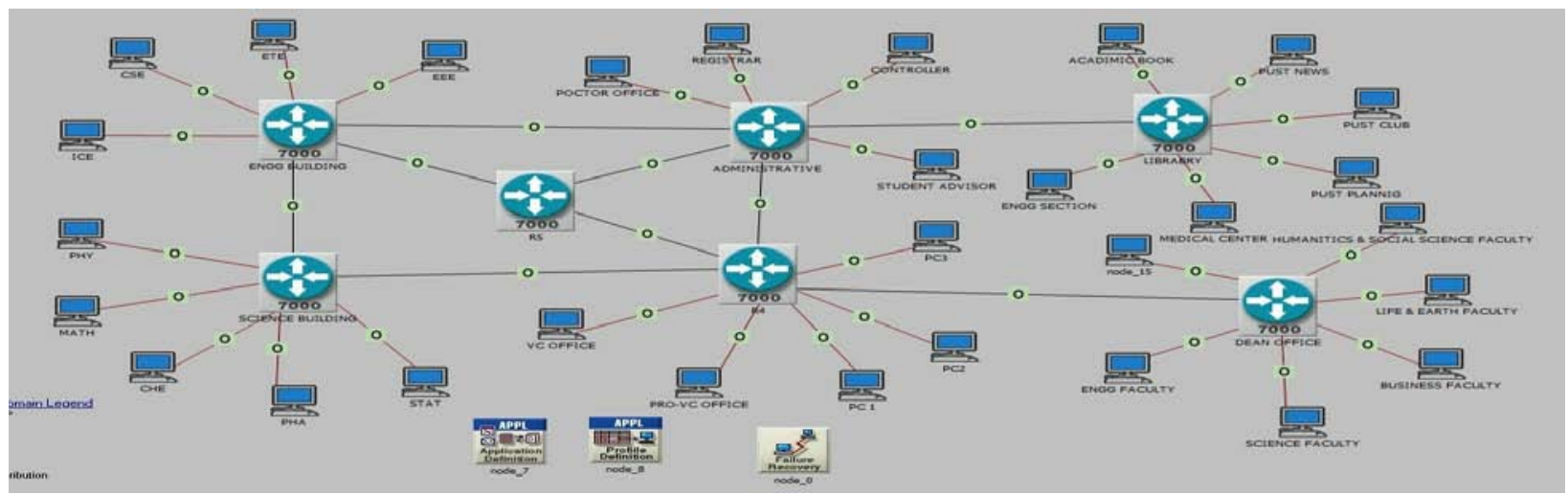

Fig.13: OSPF scenario

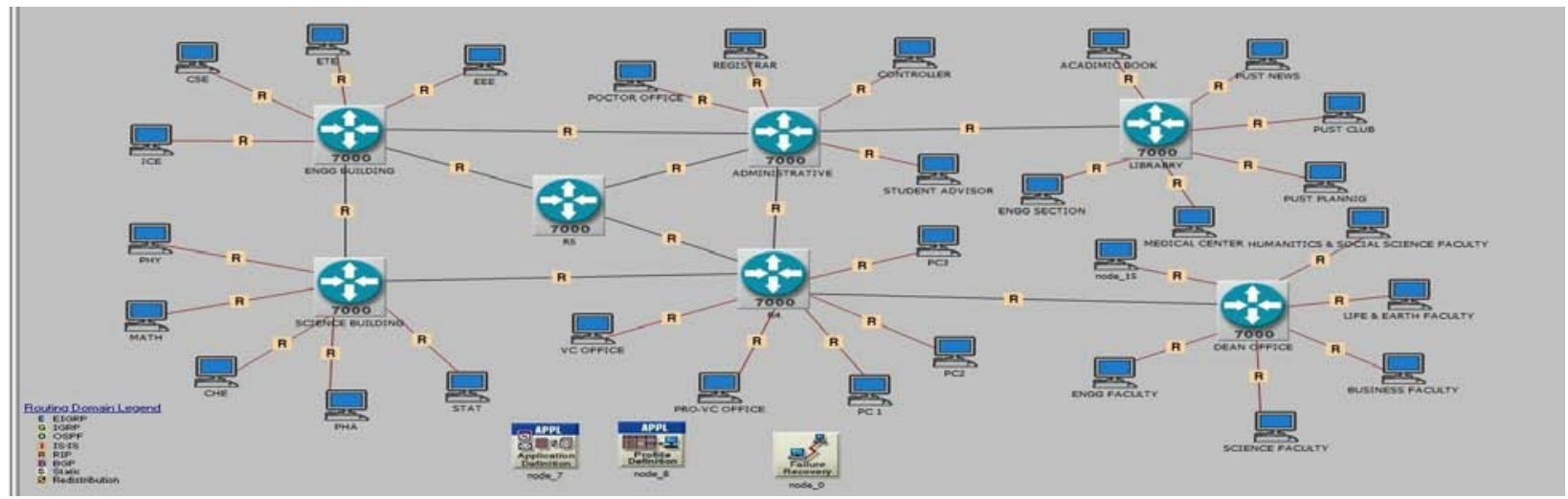

Fig. 14: RIP scenario

Fig.12 shows the scenario of EIGRP. We enable EIGRP routing protocol for all routers on the network. After configuring routing protocols, we choose individual DES statistics to select performance metrics and to measure the behavior of the routing protocol. Then we set simulation run time to 15 minutes.

Fig.13 shows the scenario of OSPF. We enable OSPF routing protocol for all routers on the network. After configuring routing protocols, we choose individual DES statistics to select performance metrics and to measure the behavior of the routing protocol. Then we set simulation run time to 15 minutes.

Fig.14 shows the scenario of RIP. We enable RIP routing protocol for all routers on the network. After configuring routing protocols, we choose individual DES statistics to select performance metrics and to measure the behavior of the routing protocol. Then we set simulation run time to 15 minutes.

\section{Results and Discussion}

Based on the above topology, we have simulated the performance of each routing protocol. We have presented a comparative analysis of EIGRP, OSPF and RIP. We have configured and run the three networks models as 1st scenario with EIGRP alone, 2nd one with OSPF alone and 3rd one with RIP concurrently. Link failure between the Director and the Engineering router has been configured in the Table. 
a) Convergence Duration

Fig.15 shows that the convergence time of EIGRP is faster than OSPF and RIP networks.

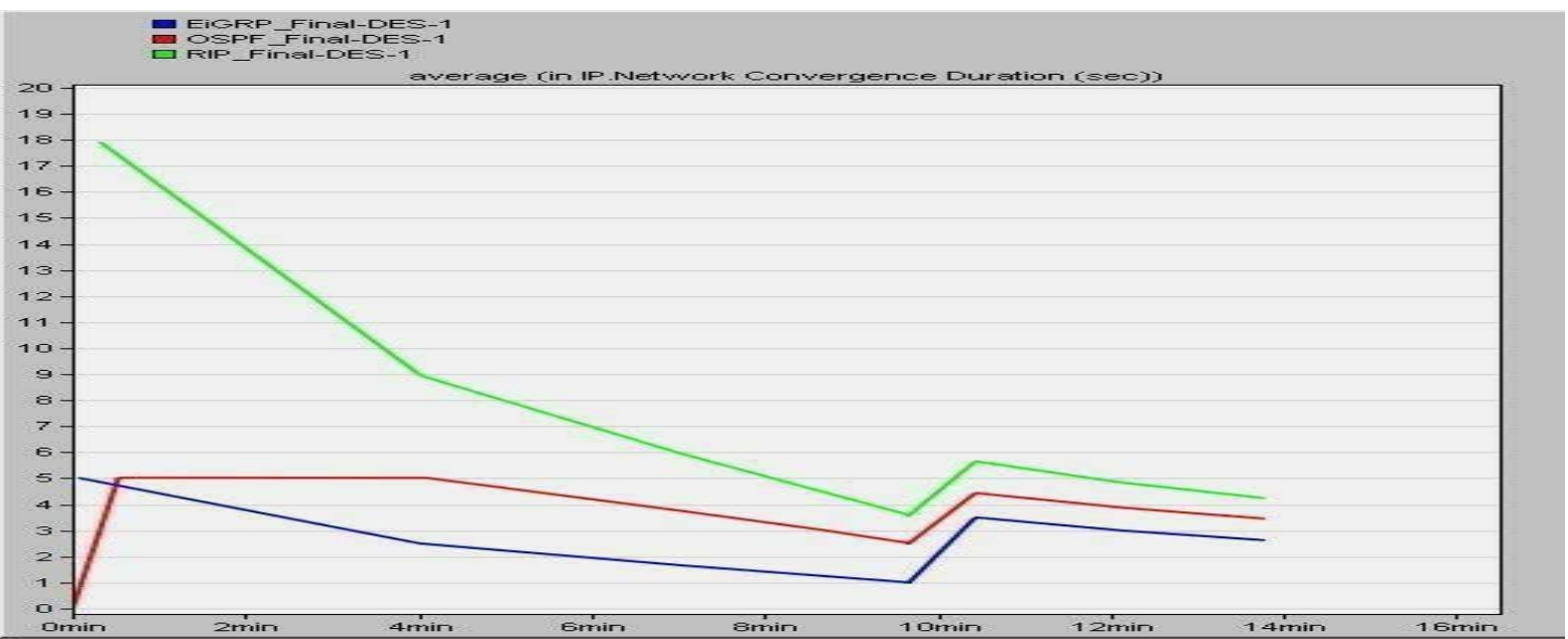

Fig. 15: Convergence duration

Because when the change occurs through the network, it detects the topology change and sends a query to the immediate neighbors to have a successor and propagates this update to all routers. The network convergence time of OSPF is slower than EIGRP and RIP networks. As the change occurred in the OSPF network, all routers within an area update the topology database by flooding LSA to the neighbors and recalculate the routing table. As a consequence, the network convergence time of OSPF is getting slower than others. Fig.15 indicates that the convergence time of EIGRP is getting decreased rapidly with the increment of the OSPF network. In contrast, the convergence time of the RIP network is slower than the OSPF network.

\section{b) Traffic sent comparison on three routing protocols}

Fig.16 shows the router traffic sent in bits/sec in three routing protocols. From the graph, the first peak is the initial traffic, the next peak is link failure, and the last peak is the link recovery in the network.

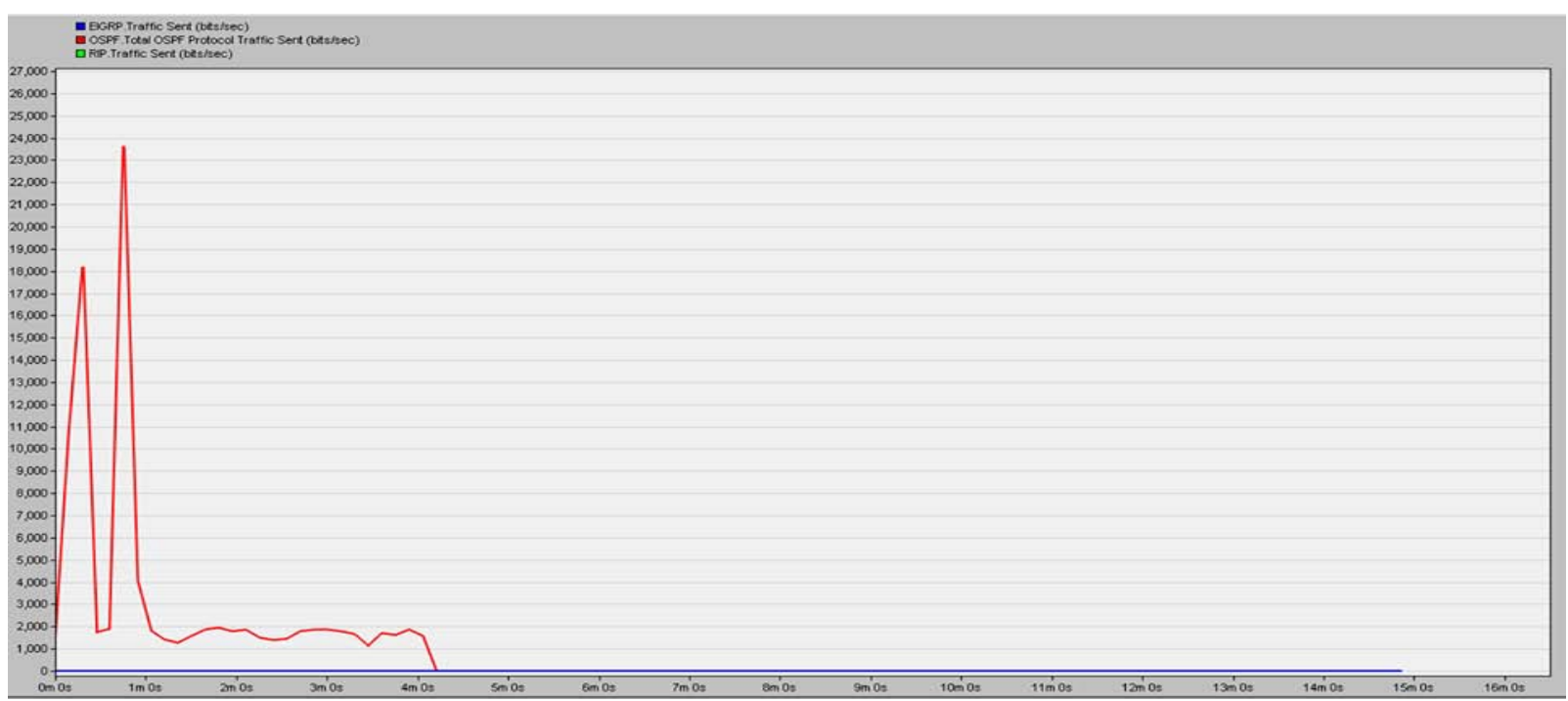

Fig. 16: Traffic sent comparison on three routing protocols

We can tell that OSPF generates the highest initial traffic since the OSPF will map out the network which requires routers to distribute a large amount of information than choosing a path. Also we note that EIGRP has the highest bandwidth efficiency, and the second one is OSPF. However, the RIP has the lowest bandwidth efficiency.

\section{Vi. Conclusion \& Future Work}

\section{a) Conclusion}

In this paper, we have designed a similar network configuration for all three routing protocols EIGRP, OSPF, and RIP by using Cisco Packet Tracer and OPNET. Then we have analyzed the performances of these protocols based on the performance metrics 
convergence duration, and traffic sent(bits/sec) to compare the difference in their performance. According to the convergence duration results, EIGRP is the fastest routing protocol among all the three protocols when initializing, failing, and recovering. OSPF is the slowest (OSPF has to let all the routers to know each other) when initializing which matches our result. According to the traffic sent (bits/sec), we can conclude that OSPF and EIGRP benefit from the bandwidth while RIP sends complete information to flood the network which wasted bandwidth. Refer to our analysis of all simulation results; we can conclude that EIGRP is the best choice for both large and small networks since it has the fastest convergence and EIGRP uses the bandwidth efficiently.

b) Future Work and difficulties

In the future, we will do some security analysis for RIP, OSPF and EIGRP. Also we can implement different topologies in terms of the number of routers and links, distance and topology type. In our work, we have analyzed for RIPv2, OSPF and EIGRP in the IPv4 environment based on OPNET. In the future, we will compare OSPFV3 and EIGRP in the IPV6 environment using OPNET.

\section{References Références Referencias}

1. Behrouz A. Forouzan, "TCP/IP Protocol Suite", McGraw-Hill Education Press. P. 269.ISBN 0-07337604-3.Retrieved on March 25, 2009.

2. http://www.cisco.com/c/en/us/support/docs/ip/enha nced-interior-gateway-routing-protocol-eigrp/16406 -eigrp-toc.html

3. "Introduction to OPNET Simulator" http://users. salleurl.edu/ zaballos/opnet_interna/pdf/OPNET\%2 OSimulato r.pdf

4. "Simulations and Tools for Telecommunications "http://www.telecomlab.oulu.fi/kurssit/521365A_tieto liikennetekniikan_simuloinnit_ja_tyokalut/Opnet_esittely_07.pdf

5. D. Sankar and D. Lancaster, "Routing Protocol Convergence Comparison using Simulation and Real Equipment". Advances in Communications, Computing, Networks and Security Volume 10, ISBN: 978-1-84102-358-8, pp186-194, 2013.

6. Hubert Pun, "Convergence Behavior of RIP and OSPF Network Protocols". Retrieved in Dec 2001.

7. K.Mirzahossein, M.Nguyenand S.Elmasre "Analysis of RIP, OSPF, and EIGRP Routing Protocols using OPNET". Retrieved in 2013 Internet: http://www.sfu.ca/ mtn9/427_Report.pdf

8. B. Wu, "Simulation Based Performance Analysis on RIPV2, EIGRP and OSPF Using OPNET". Retrieved on Mar 15, 2013

9. Ayub, N., F. Jan, et al. (2011). "Performance Analysis of OSPF and EIGRP Routing Protocols with Respect to the Convergence" European Journal of Scientific Research 61(3):434-447.
10. Kurose, J. F. and K. W. Ross (2010). Computer networking a top-down approach. Boston, Addison-Wesley. 